\title{
Absorption, refraction and scattering retrieval with an edge-illumination-based imaging setup
}

\author{
Marco Endrizzi*and Alessandro Olivo \\ Department of Medical Physics and Bioengineering \\ University College London \\ Gower Street, WC1E 6BT London, United Kingdom
}

\begin{abstract}
We have recently developed a new method based on edge-illumination for retrieving a three-image representation of the sample. A minimum of three intensity projections is required in order to retrieve the transmission, refraction and ultra-small-angle scattering properties of the sample. Here we show how the method can be adapted for particular cases in which some degree of a priori information about the sample might be available, limiting the number of required projections to two. Moreover, an iterative algorithm to correct for non-ideal optical elements is proposed and tested on numerical simulations, and finally validated on experimental data.
\end{abstract}

\section{Introduction}

X-ray Phase-Contrast Imaging (XPCI) overcomes limitations of conventional radiography by introducing sensitivity also to the phase shifts suffered by the X-ray beam as it travels through the sample [1]. Applications of XPCI are extremely vast, ranging from biological and medical to security inspection and materials science. Several approaches exist for obtaining phase-contrast images in the X-ray regime, using large-scale synchrotron facilities and more compact X-ray-tube-based equipment [2-17]. The imaging methods, their developments and applications can also be found in recent reviews on the subject [18-21]. Edge-illumination [9] and its area imaging counter part coded-aperture [22] are XPCI techniques capable of quantitative amplitude and phase retrieval [23]. These methods were implemented with synchrotron radiation [9] as well as with rotating anode [22; 23] and microfocal [24] X-ray tubes. The retrieval of ultrasmall-angle X-ray scattering in the hard X-rays regime was also recently developed for an edge-illumination set-up [25]. This approach to quantitative XPCI was proven to work under extremely weak coherence conditions, and uses the full broadband spectrum typically produced by an X-ray tube. It simultaneously produces three representations of the sample that can provide complementary information for better identification and discrimination between materials and

*m.endrizzi@ucl.ac.uk 
types of tissues [26-28]. The work presented here builds on these results, focusing on the expansion of the method to include system imperfections that can be encountered in practical situations and the exploitation of a priori knowledge that might be available about the sample. Both analytical formulae and an iterative algorithm are proposed and tested on numerical simulations. Furthermore, it is shown how a simulation study for the noise propagation from the intensity projections to the retrieved images enables the optimization of the data acquisition scheme. Finally, the results are applied to experimental data, and a discussion of possible limitations of the method is presented.

\section{Methods}

A typical experimental set-up for edge-illumination consists of an absorbing slit, shaping the X-ray beam before it reaches the sample, aligned with an absorbing edge, placed just in font of the detector pixels. This second absorbing element serves as an analyser and converts fine angular deflections of the beam into intensity variations recorded by the detector (Fig. 1(a)). This can be extended to perform area imaging by stacking together a series of pre-sample apertures and aligning them with the corresponding edges and detector pixels (Fig. 1(b)). In a typical laboratory experiment, relatively large sources and short distances

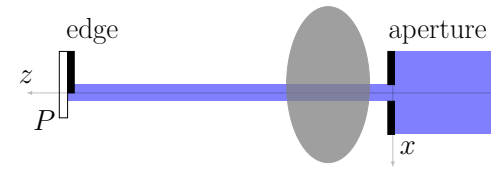

(a)

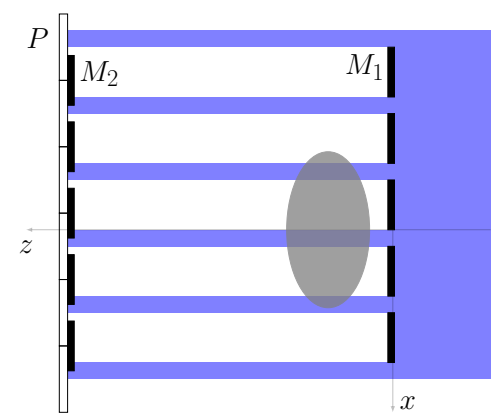

(b)

Figure 1: Typical experimental setups for (a) edge illumination and (b) codedaperture XPCI. (a) The X-ray beam, coming from the right-hand side, is shaped by an absorbing slit and it is analysed with an absorbing edge just before the detector pixel $P$. (b) This concept is adapted for area imaging by stacking together a series or apertures $M_{1}$ matching the relative apertures on the detector mask $M_{2}$.

are used, and the image formation can be described on a pixel-by-pixel basis using geometrical optics. The image recorded by such a set-up can be described 
in the following way [25]:

$$
\frac{I(x)}{I_{0}}=(L * O)\left(x-\Delta x_{R}\right) t
$$

where $I_{0}$ is the intensity going trough the pre-sample aperture, $L$ is the illumination function characteristic of the imaging system, $*$ denotes convolution and the scattering distribution induced by the sample is represented by $O$. $\Delta x_{R}=-\Delta \theta_{R} z_{o d}$ is the beam shift due to the sample-induced refraction $\Delta \theta_{R}$ $\left(z_{o d}\right.$ is the distance between the object and the detector) and $t$ is the fraction of the total intensity transmitted through the sample. The functions $L$ and $O$ can be rewritten as a sum of Gaussian functions, and Eq. 1 takes the general form [25]:

$$
\frac{I(x)}{I_{0}}=t \sum_{m} \sum_{n} A_{m n} \exp \left[-\frac{\left(x-\mu_{m n}\right)^{2}}{2 \sigma_{m n}^{2}}\right]
$$

where $\mu_{m n}=\mu_{m}+\mu_{n}, \sigma_{m n}^{2}=\sigma_{m}^{2}+\sigma_{n}^{2}, A_{m n}=A_{m} A_{n}\left(1 / \sqrt{2 \pi \sigma_{m n}^{2}}\right)$. The functions representing the illumination function and the scattering were expanded as follows: $L(x)=\sum_{n=1}^{N}\left(A_{n} / \sqrt{2 \pi \sigma_{n}^{2}}\right) \exp \left[-\left(x-\mu_{n}\right)^{2} / 2 \sigma_{n}^{2}\right]$ and $O(x)=\sum_{m=1}^{M}\left(A_{m} / \sqrt{2 \pi \sigma_{m}^{2}}\right) \exp \left[-\left(x-\mu_{m}\right)^{2} / 2 \sigma_{m}^{2}\right]$, respectively.

Analytical inversion formulae were proposed for Eq. 2 by acquiring three projection images with the displacement of the masks set to $x_{2}=0$ and $x_{1}=$ $-x_{3}[25]$

$$
I_{i}=t \frac{A_{M N}}{\sqrt{2 \pi \sigma_{M N}^{2}}} \exp \left[-\frac{\left(x_{i}-\Delta x_{R}\right)^{2}}{2 \sigma_{M N}^{2}}\right], \quad i=1,2,3
$$

from which $t, \Delta x_{R}$ and $\sigma_{M}^{2}$ can be retrieved:

$$
\left\{\begin{aligned}
t & =\frac{2 x_{1}}{A_{M N}} \sqrt{\frac{\pi}{D+C}} I_{2} \exp \left[\frac{1}{2^{4}} \frac{(D-C)^{2}}{D+C}\right] \\
\Delta x_{R} & =\frac{x_{1}}{2} \frac{D-C}{D+C} \\
\sigma_{M}^{2} & =\frac{2 x_{1}^{2}}{D+C}-\sigma_{N}^{2}
\end{aligned}\right.
$$

where $C=-2 \ln \left(I_{1} / I_{2}\right)$ and $D=-2 \ln \left(I_{3} / I_{2}\right)$, and a single Gaussian term was used for $L(x)$ and for $O(x)$.

These solutions can be specialized when a priori information about the sample is available. If it is known a priori that the sample under investigation presents negligible refraction $\left(\Delta x_{R}=0\right)$, the transmission and the scattering image can be retrieved by using only two projections

$$
\left\{\begin{aligned}
\sigma_{M}^{2} & =\frac{x_{1}^{2}}{C}-\sigma_{N}^{2} \\
t & =\frac{I_{2}}{A_{M N}} \sqrt{\frac{2 \pi x_{1}^{2}}{C}} .
\end{aligned}\right.
$$

Similarly, for the case in which $\sigma_{M}=0$ (negligible scattering) transmission and refraction images can be retrieved from two projection images as

$$
\left\{\begin{aligned}
\Delta x_{R} & =\frac{\sigma_{N}^{2} E}{x_{1}} \\
t & =\frac{I_{1}}{A_{M N}} \sqrt{2 \pi \sigma_{N}^{2}} \exp \left[\frac{\left(x_{1}^{2}-E \sigma_{N}^{2}\right)^{2}}{2 x_{1}^{2} \sigma_{N}^{2}}\right]
\end{aligned}\right.
$$


where $E=(1 / 2) \log \left(I_{1} / I_{3}\right)$.

In an actual experiment, it can occur that the illumination function appears with an offset, i.e. when the masks are completely mismatched one with respect to the other, an intensity different from zero is recorded at the detector [29]. This could be due, for example, to a non-completely absorbing mask that would let part of the radiation trough the opaque sectors. In order to account for this, the illumination function needs to be represented with the addition of a constant term $c$. For a relative displacement of the masks $x_{i}$, the recorded image becomes

$$
I_{i}=t \frac{A_{M N}}{\sqrt{2 \pi \sigma_{M N}^{2}}} \exp \left[-\frac{\left(x_{i}-\Delta x_{R}\right)^{2}}{2 \sigma_{M N}^{2}}\right]+t c
$$

where $c$ is a constant, characteristic of the setup, that can be known from an experimental measurement of $L$. If also $t$ was known, it would be possible to correct the measurements $I_{i}$ by the amount $t c$ and reduce to the problem in Eq. 3. This is not usually the case, as $t$ depends on the sample itself; however, Eq. 7 can be solved by means of an iterative procedure. We start with the initial guess that $c=0$ and retrieve $t, \Delta x_{R}$ and $\sigma_{M}^{2}$ with Eq. 4 by using the three projection images acquired at $x_{2}=0, x_{1}$ and $x_{3}$. This gives an initial estimation for $t$ that can be used to correct $I_{i}$, and the process is iterated. More formally, we iterate over $k=0 \ldots K$ the set of equations:

$$
\left\{\begin{aligned}
t^{k+1} & =\frac{2 x_{1}}{A_{M N}} \sqrt{\frac{\pi}{D^{k}+C^{k}}} I_{2} \exp \left[\frac{1}{2^{4}} \frac{\left(D^{k}-C^{k}\right)^{2}}{D^{k}+C^{k}}\right] \\
\Delta x_{R}^{k+1} & =\frac{x_{1}}{2} \frac{D^{k}-C^{k}}{D^{k}+C^{k}} \\
\sigma_{M}^{2}{ }^{k+1} & =\frac{2 x_{1}^{2}}{D^{k}+C^{k}}-\sigma_{N}^{2}
\end{aligned}\right.
$$

where

$$
\left\{\begin{array}{l}
C^{k}=-2 \ln \left[\left(I_{1}-I_{r}\right) /\left(I_{2}-I_{r}\right)\right] \\
D^{k}=-2 \ln \left[\left(I_{3}-I_{r}\right) /\left(I_{2}-I_{r}\right)\right]
\end{array}\right.
$$

and $I_{r}=t^{k} c\left[1-\left(1 / 2^{k}\right)\right]$. Together with the iterative procedure, a stopping criterion is required in order to establish the actual value of $K$. We decide when to stop the iteration by monitoring the relative change in the retrieved images; for example, by referring to $t$, we calculate

$$
L^{k}=\sqrt{\frac{1}{N} \sum_{i j}\left(t_{i j}^{k-1}-t_{i j}^{k}\right)^{2}}
$$

where $(i, j)$ run over the image pixels, and $N$ is their total number. When the iteration does not change the retrieved images any more, the loop is interrupted. We note also that this approach can be analogously applied in the special cases discussed above (Eq. 5 and Eq. 6).

The numerical phantoms used in the simulations consist of $512 \times 512$ pixels images with $t, \Delta \theta$ and $\sigma_{M}$ in the intervals $[0.5,1],[-5,5] \mu \mathrm{rad}$ and $[0,15]$ $\mu \mathrm{rad}$, respectively. They are composed of a vertical wedge (absorbing, refracting and scattering) and a cylinder (absorbing and refracting) superimposed one to the other (see also Fig. 4(d), 4(e) and 4(f)). With reference to Fig. 1, the image plane is $(x, y)$ and apertures are oriented vertically, along the $y$ direction. The detector is modelled as an ideal single-photon-counter and the noise is simulated by using Poisson statistics. The parameters for the illumination 
functions are extracted from the empirical measurement of $L$ (see also below in Fig. 2(b)). The masks parameters and the geometry are the same we used for the experiment, which is described in the following.

The experimental set-up consists of a Mo target, rotating anode X-ray tube (Rigaku MM007) and an amorphous Selenium flat panel (Anrad SMAM) with a pixel pitch of $p_{3}=85 \mu \mathrm{m}$. The masks, manufactured to our design by Creatv Microtech (Potomac, MD), were aligned with a stack of Newport (ILS150, MFA and SR50) and Kohzu (SA07A-RM) stages. The pre-sample mask had a pitch of $p_{1}=66.8 \mu \mathrm{m}$ and apertures of $b_{1}=12 \mu \mathrm{m}$. The detector mask had a pitch of $p_{2}=83.5 \mu \mathrm{m}$ and apertures of $b_{2}=20 \mu \mathrm{m}$. The absorbing material was gold, approximately $30 \mu \mathrm{m}$ thick, on a graphite substrate. The field of view was $4.8 \times 4.8 \mathrm{~cm}$. The source to detector distance was $2 \mathrm{~m}$ and the object to detector distance $z_{o d}=40 \mathrm{~cm}$. The $I_{1}$ and $I_{3}$ images where acquired using a misalignment $x_{1}=12 \mu \mathrm{m}$. The phantom was composed of two step-wedges, one made of five layers of paper $(80 \mu \mathrm{m}$ each) and the other of five layers of polypropylene plastic $(800 \mu \mathrm{m}$ each).

\section{Results}

The illumination function $L \equiv B_{1} * S * B_{2}[25]$, where $B_{1,2}$ are rectangular functions and $S$ is the source distribution projected at the detector plane, can be represented with a Gaussian function when an extended source is used. $b_{1,2}$ indicate the pre-sample and the detector aperture widths, respectively. The validity of this approximation depends on the particular values of the aperture widths, the source size and the relative distances between them. For the geometry of our experimental set-up, the maximum error $\left\{\left|L(x)-L_{f}(x)\right|\right\}_{\text {max }}$ between $L(x)$ and its Gaussian fit $L_{f}(x)$ was calculated for several sets of apertures and source dimensions. The results are reported in Fig. 2(a): the approximation is generally acceptable, and becomes increasingly reliable when a larger source is used. Describing $B_{1}$ and $B_{2}$ with rectangular functions requires that the illumination function reaches zero on the tails. This is not always the case for an actual experiment and often an offset, typically between $5-30 \%$, is observed. When this is the case, $L$ can be represented by a Gaussian term plus a constant term $c$. In Fig. 2(b) the illumination function measured on experimental data is plotted along with its fit by using a Gaussian term plus offset. The ideal illumination function, calculated as $L=B_{1} * S * B_{2}$, and its single-Gaussian fit are also shown in the same panel. For values of the illumination function above 0.4, a single Gaussian term still provides a good approximation; this however gradually loses accuracy as the illumination levels are diminished.

The iterative solution, for the retrieval of transmission, refraction and scattering, was tested on the numerical phantom for $c$ values of 0.2 and 0.3 . The error $e_{k}=\sqrt{(1 / N) \sum_{i j}\left(f_{i j}-f_{i j}^{k}\right)^{2}}$, where $f_{i j}$ is the known exact distribution of values and $f_{i j}^{k}$ is the one retrieved at the $k$-th iteration, is plotted against the number of iterations in Fig. 3. The dashed lines indicates the error between the exact and the retrieved solutions when $c=0$, and the three channels are represented with different colours: $t$ blue, $\Delta x_{R}$ green and $\sigma_{M}^{2}$ red. The algorithm converges to the exact solution within a few tens of iterations for $c=0.2$ and less than two hundreds for $c=0.3$. As mentioned, the same correction algorithm 


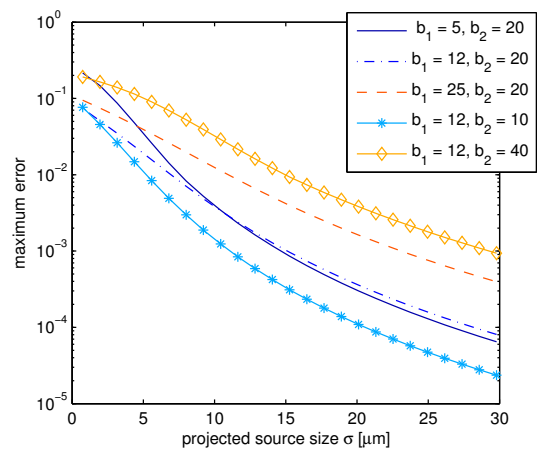

(a)

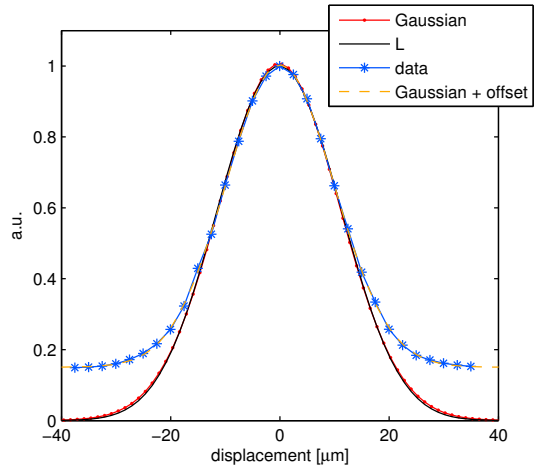

(b)

Figure 2: (a) maximum error caused by the use of a Gaussian term to analytically describe the illumination function $L$ for various $b_{1}$ and $b_{2}$ parameters, as a function of the projected source size. The numbers indicated in the legend are in $\mu \mathrm{m}$. (b) graphical comparison between an ideal $L$ (for $b_{1}=12 \mu \mathrm{m}, b_{2}=20 \mu \mathrm{m}$ and source width $70 \mu \mathrm{m}$ ) and its Gaussian fit, and between an experimentally measured $L$ and its Gaussian plus offset fit.

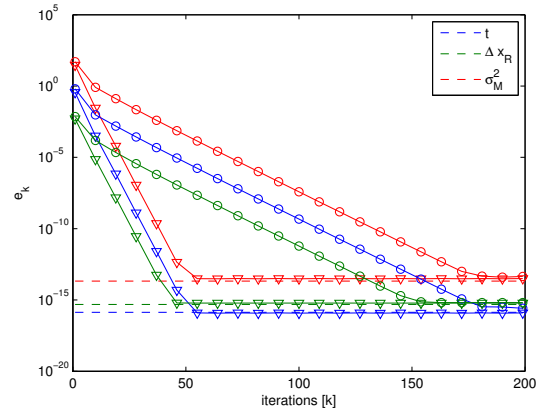

(a)

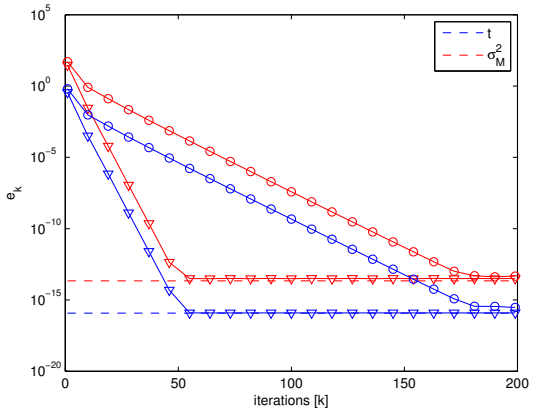

(b)

Figure 3: Convergence of the iterative solution for offset illumination functions, $c=0.2$ (triangles) and $c=0.3$ (circles). (a) three-image retrieval in the general case and (b) two-image retrieval for the special case $\Delta x_{R}=0$. 
can be applied in the particular cases described above, where the acquisition of only two images is required. As an example, the simulated $I_{i}$ measurements and the retrieved images are shown in Fig. 4. In this case, an offset $c=0.3$ was used and the images were retrieved with 200 iterations. A smaller $c$ parameter corresponds to a smaller number of iterations required to achieve the desired accuracy, which is typically reached with a few seconds of computing time on a standard desktop machine $(2.33 \mathrm{GHz})$.

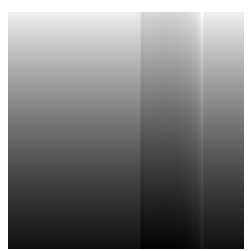

(a)

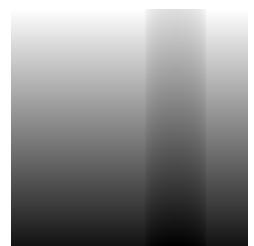

(d)

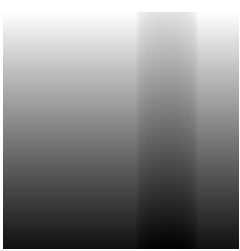

(b)

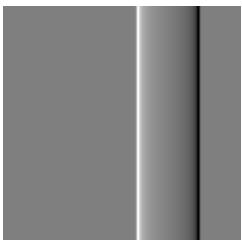

(e)

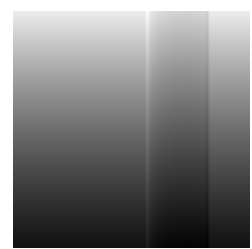

(c)

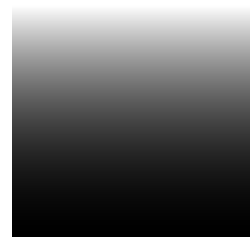

(f)

Figure 4: Example of simulated projection images $I_{1}$ (a), $I_{2}$ (b) and $I_{3}$ (c), with the pre-sample mask positioned respectively on the left-, top and righthand side of the illumination function. The transmission (d), refraction (e) and scattering (f) are retrieved from a simulation with $c=0.3$ offset and performing 200 iterations.

For evaluating the propagation of the noise from the projection images to the retrieved ones, the data acquisition was simulated using Eq. 3 and assuming an ideal single-photon-counting detector. The relative displacement $x_{1}$ between the masks was changed while keeping constant the number of photons transmitted through the pre-sample aperture. Noise was added to the images according to Poisson's statistics. The results obtained using $10^{4}$ photons are shown in Fig. 5. The minimum error $e$ is reached at $x_{1}=\{11.6,12.6,19.4\} \mu \mathrm{m}$ for $t, \Delta x_{R}$ and $\sigma_{M}^{2}$ respectively. The possible effect of photon statistics on the above set of $x_{1}$ values was investigated by repeating this set of simulations while varying the total number of photons allowed through the sample aperture. No significant difference was found, for a number of photons ranging between 10 and $10^{6}$ the minima were always found to be within $0.5 \mu \mathrm{m}$ from the positions stated above. The minima are reached at different displacements for the three parameters extracted. This is the result of the interplay between the accuracy in the measurement of absorption, dominated by the detected number of photons, of refraction, for which the maximum change in signal for a given $\Delta x_{R}$ occurs where the slope of the illumination function is maximum and of scattering for which one would aim to minimize the amount of unscattered radiation reaching the active area of the pixel.

Finally, the method was applied to the experimental projection images ac- 

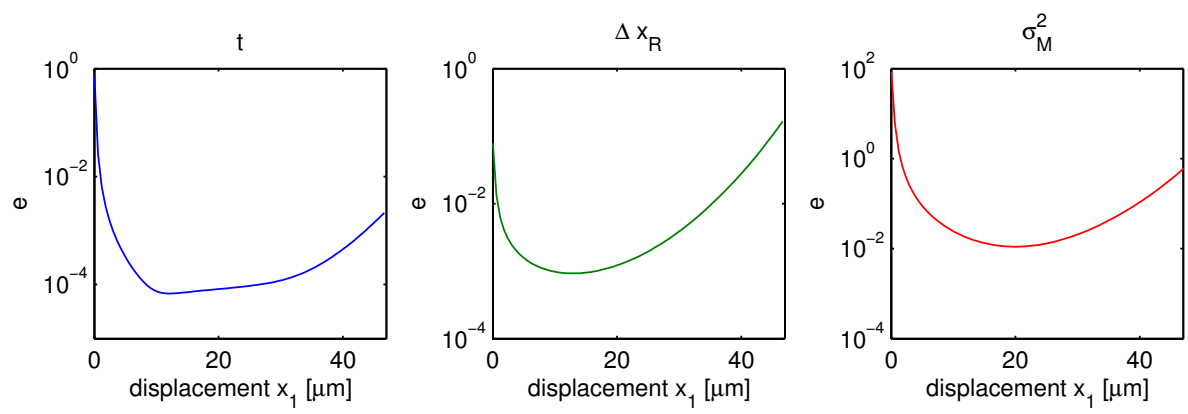

Figure 5: Noise propagation to the retrieved images. The number of photons $\left(10^{4}\right)$ transmitted through the pre-sample aperture is kept constant while changing the relative displacement $x_{1}$ between the masks. Optimum displacements are $x_{1}=\{11.6,12.6,19.4\} \mu \mathrm{m}$ for $t, \Delta x_{R}$ and $\sigma_{M}^{2}$ respectively.

quired operating the source at $35 \mathrm{kVp}$. Transmission, refraction and scattering were retrieved by using the sets of equations 8 and 9 , and the results are shown in Fig. 6. The paper step-wedge is on the left-hand side while the polypropylene one is on the right. It can be observed how polypropylene shows contrast only in the absorption image (except for its edges), while paper exhibits some degree of contrast in all the three channels. The iterative algorithm correctly compensates for the excess of intensity which is observed in the projection images due to the fact that the illumination function is offset from zero. In the panels Fig. 6(d) and Fig. 6(d), the transmission and scattering images are shown for the two-image retrieval that assumes $\Delta x_{R}=0$ (Eq. 5), for which the same iterative correction scheme of Eq. 9 has been applied. The artefacts along the vertical edges are due to the fact that refraction is assumed to be negligible, which results in a poor approximation for those parts of the sample. The number of iterations used for the three-image case was 280 , which was estimated by looking at the relative change in the retrieved images at each iteration. An example of how this kind of plot enables stopping the iterative algorithm is shown in Fig. 7 .

A remark on the possible limitations of this iterative approach is related to beam hardening. The illumination function offset can be caused by a noncomplete absorption of the incoming radiation in the gold substrate of the detector mask. This problem becomes more severe with increasing X-ray energy since, for a given gold thickness, a larger fraction of the total intensity will reach the detector passing through the gold layer. This introduces a sampledependent type of artefact which is demonstrated by the experimental images in Fig. 8. These images were retrieved from projections acquired operating the source at $45 \mathrm{kVp}$, and therefore introducing a harder component in X-ray beam with respect to the previous case. A small residual signal can be observed in the scattering image (Fig. 8(c)), stronger where the polypropylene is thicker. This can be explained by the fact that the spectrum emerging from a thicker layer of absorber is harder, and will therefore give a larger contribution to the offset of the illumination function. Unfortunately this effect is sample- (and position-) dependent and cannot be completely corrected with the algorithm proposed here. Depending on the experimental conditions and on the quality 


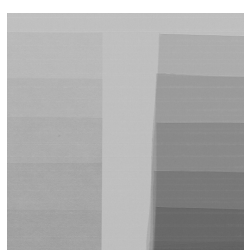

(a)

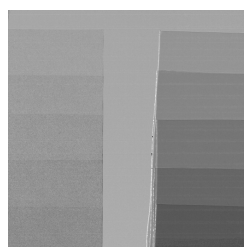

(d)

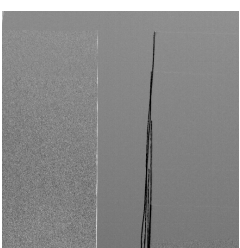

(b)

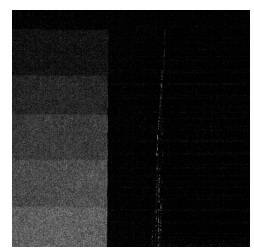

(c)

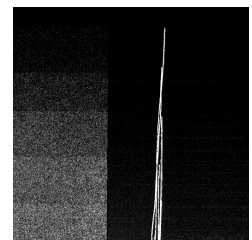

(e)

Figure 6: Retrieval by using Eq. 8 and 9 on experimental projection images acquired at $35 \mathrm{kVp}$. (a) transmission, (b) refraction and (c) scattering of the phantom. The sensitivity is in the horizontal direction, the bright and dark vertical stripes correspond to the paper and polypropylene edges, respectively. The paper step-wedge is on the left-hand side while the polypropylene one is on the right. Retrieval by using Eq. 5 and the iterative corrections of Eq. 9: (d) transmission and (e) scattering.

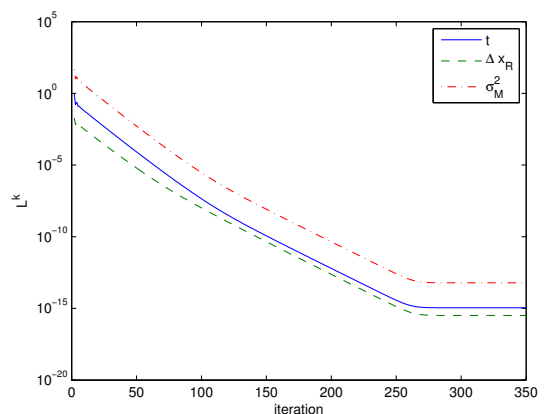

Figure 7: Stopping criterion for the experimental case. The number of iterations was estimated to be 280 , after which the retrieved image does not change any more. 


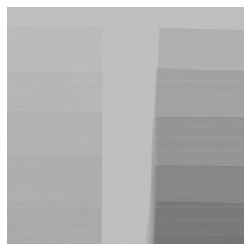

(a)

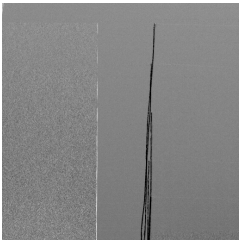

(b)

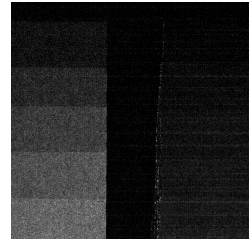

(c)

Figure 8: Retrieval by using Eq. 8 and 9 on experimental projection images acquired at $45 \mathrm{kVp}$. (a) transmission, (b) refraction and (c) scattering of the phantom. The paper step-wedge is on the left-hand side while the polypropylene one is on the right.

of the available optical elements, this could be a potential source of inaccuracy, for example when the different signals are combined in order to obtain material-specific signatures.

\section{Conclusions}

A recently proposed method for multi-modal imaging with a laboratory set-up based on edge illumination was further developed and extended. The retrieval was specialized to particular cases in which some a priori knowledge on the sample is available, potentially limiting the minimum number of projection images to two. An iterative algorithm to correct the inaccuracies in the retrieval related to the presence of an offset in the illumination function was developed and tested both on numerical simulations and experimentally on a custom made phantom. The propagation of noise from the intensity projections to the retrieved images was evaluated by means of numerical simulations, which enabled the optimization of the data acquisition procedure. A brief discussion on the possible limitations of the method, related to the local hardening of the X-ray beam, was finally presented.

\section{Acknowledgments}

This work was supported by the UK Engineering and Physical Sciences Research Council Grant EP/I021884/1. ME is supported by Marie Curie Career Integration Grant within the Seventh Framework Programme of the European Union PCIG12-GA-2012-334056.

\section{References}

[1] R. Fitzgerald. Phase-sensitive x-ray imaging. Physics Today, 53(7):23-26, 2000.

[2] U. Bonse and M. Hart. An x-ray interferometer. Applied Physics Letters, 6:155-156, 1965. 
[3] K. Goetz, E. Foerster, P. Zaumseil, M. P. Kalashnikov, I. A. Mikhailov, G. V. Sklizkov, and S. I. Fedotov. Measurements of the parameters of shell targets for laser thermonuclear fusion using an X-ray schlieren method. Kvantovaia Elektronika Moscow, 6:1037-1042, 1979.

[4] A. Snigirev, I. Snigireva, V. Kohn, S. Kuznetsov, and I. Schelokov. On the possibilities of x-ray phase contrast microimaging by coherent high-energy synchrotron radiation. Rev. Sci. Instrum., 66(12):5486-5492, 1995.

[5] T. J. Davis, D. Gao, T. E. Gureyev, A. W. Stevenson, and S. W. Wilkins. Phase-contrast imaging of weakly absorbing materials using hard X-rays. Nature, 373:595-598, 1995.

[6] V. N. Ingal and E. A. Beliaevskaya. X-ray plane-wave topography observation of the phase contrast from a non-crystalline object. J. Phys. D Appl. Phys., 28:2314-2317, 1995.

[7] S. W. Wilkins, T. E. Gureyev, D. Gao, A. Pogany, and A. W. Stevenson. Phase-contrast imaging using polychromatic hard x-rays. Nature, 384:335$338,1996$.

[8] D. Chapman, W. Thomlinson, R. E. Johnston, D. Washburn, E. Pisano, N. Gmür, Z. Zhong, R. Menk, F. Arfelli, and D. Sayers. Diffraction enhanced x-ray imaging. Physics in Medicine and Biology, 42:2015-2025, 1997.

[9] A. Olivo, F. Arfelli, G. Cantatore, R. Longo, R. H. Menk, S. Pani, M. Prest, P. Poropat, L. Rigon, G. Tromba, E. Vallazza, and E. Castelli. An innovative digital imaging set-up allowing a low-dose approach to phase contrast applications in the medical field. Medical Physics, 28:1610-1619, 2001.

[10] C. David, B. Nohammer, H. H. Solak, and E. Ziegler. Differential x-ray phase contrast imaging using a shearing interferometer. Applied Physics Letters, 81(17):3287-3289, 2002.

[11] L. Rigon, H.-J. Besch, F. Arfelli, R.-H. Menk, G. Heitner, and H. PlothowBesch. A new DEI algorithm capable of investigating sub-pixel structures. J. Phys. D Appl. Phys., 36(26):A260000-A112, 2003.

[12] E. Pagot, P. Cloetens, S. Fiedler, A. Bravin, P. Coan, J. Baruchel, J. Härtwig, and W. Thomlinson. A method to extract quantitative information in analyzer-based x-ray phase contrast imaging. Applied Physics Letters, 82:3421, 2003.

[13] M. N. Wernick, O. Wirjadi, D. Chapman, Z. Zhong, N. P. Galatsanos, Y. Yang, J. G. Brankov, O. Oltulu, M. A. Anastasio, and C. Muehleman. Multiple-image radiography. Physics in Medicine and Biology, 48:3875$3895,2003$.

[14] F. Pfeiffer, T. Weitkamp, O. Bunk, and C. David. Phase retrieval and differential phase-contrast imaging with low-brilliance X-ray sources. Nature Physics, 2:258-261, 2006. 
[15] Z.-F. Huang, K.-J. Kang, L. Zhang, Z.-Q. Chen, F. Ding, Z.-T. Wang, and Q.-G. Fang. Alternative method for differential phase-contrast imaging with weakly coherent hard x rays. Phys. Rev. A, 79:013815, 2009.

[16] K. S. Morgan, D. M. Paganin, and K. K. W. Siu. Quantitative x-ray phase-contrast imaging using a single grating of comparable pitch to sample feature size. Optics Letters, 36(1):55-57, 2011.

[17] D. Pelliccia and D. M. Paganin. Multi-modal hard x-ray imaging with a laboratory source using selective reflection from a mirror. Biomedical Optics Express, 5(4):1153-1159, 2014.

[18] Y. Liu, J. Nelson, C. Holzner, J. Andrews, and P. Pianetta. Recent advances in synchrotron-based hard x-ray phase contrast imaging. Journal of Physics D: Applied Physics, 46(49):494001, 2013.

[19] P. Coan, A. Bravin, and G. Tromba. Phase-contrast x-ray imaging of the breast: recent developments towards clinics. Journal of Physics D: Applied Physics, 46(49):494007, 2013.

[20] P. Suortti, J. Keyriläinen, and W. Thomlinson. Analyser-based x-ray imaging for biomedical research. Journal of Physics D: Applied Physics, 46(49):494002, 2013.

[21] A. Bravin, P. Coan, and P. Suortti. X-ray phase-contrast imaging: from pre-clinical applications towards clinics. Physics in Medicine and Biology, 58(1):R1, 2013.

[22] A. Olivo and R. Speller. A coded-aperture technique allowing x-ray phase contrast imaging with conventional sources. Applied Physics Letters, 91(7):074106, 2007.

[23] P. R. Munro, K. Ignatyev, R. D. Speller, and A. Olivo. Phase and absorption retrieval using incoherent X-ray sources. Proc. Natl. Acad. Sci. USA, 109(35):13922-13927, 2012.

[24] M. Endrizzi, F. A. Vittoria, P. C. Diemoz, R. Lorenzo, R. D. Speller, U. H. Wagner, C. Rau, I. K. Robinson, and A. Olivo. Phase-contrast microscopy at high x-ray energy with a laboratory setup. Optics Letters, 39(11):3332$3335,2014$.

[25] M. Endrizzi, P. C. Diemoz, T. P. Millard, J. L. Jones, R. D. Speller, I. K. Robinson, and A. Olivo. Hard x-ray dark-field imaging with incoherent sample illumination. Applied Physics Letters, 104(2):024106, 2014.

[26] S. Schleede, F. G. Meinel, M. Bech, J. Herzen, K. Achterhold, G. Potdevin, A. Malecki, S. Adam-Neumair, S. F. Thieme, F. Bamberg, K. Nikolaou, A. Bohla, A. O. Yildirim, R. Loewen, M. Gifford, R. Ruth, O. Eickelberg, M. Reiser, and F. Pfeiffer. Emphysema diagnosis using x-ray dark-field imaging at a laser-driven compact synchrotron light source. Proc. Natl. Acad. Sci. USA, 109(44):17880-17885, 2012.

[27] Z. Wang and M. Stampanoni. Quantitative x-ray radiography using grating interferometry: a feasibility study. Physics in Medicine and Biology, 58(19):6815, 2013. 
[28] Z. Wang, N. Hauser, G. Singer, M. Trippel, R. A. Kubik-Huch, C. W. Schneider, and M. Stampanoni. Non-invasive classification of microcalcifications with phase-contrast x-ray mammography. Nature Communications, 5,2014 .

[29] P. R. Munro, C. K. Hagen, M. B. Szafraniec, and A. Olivo. A simplified approach to quantitative coded aperture x-ray phase imaging. Optics Express, 21(9):11187-11201, 2013. 